\title{
Research and Implementation of UAV Remote Sensing Network Dispatch Management method Based on Workflow
}

\author{
Wenxin Tian, Chaoliang Wang, Shuai Dou, Chuanrong $\mathrm{Li}^{+}$ \\ Key Laboratory of Quantitative Remote Sensing Information Technology, Academy of Opto-Electronics, \\ Chinese Academy of Sciences, Beijing, China
}

\begin{abstract}
The paper established an emergency dispatching management system for the UAV remote sensing network based on the JBPM workflow engine. The system realized the overall lifecycle management of the emergency dispatching management system for the UVA remote sensing network, providing users with an intuitive environment which is convenient to operate and maintain. Firstly, this paper introduced the basic concepts and framework of JBPM. Then, combining Petri nets theory, it established a hierarchical model of emergency scheduling management task, and validated the correctness of the model. Finally, an emergency response dispatch management system was established, achieving the purpose of lifecycle management of emergency response task. The system deployed and applied successfully in the validation task of UAV remote sensing network. It operates for long hours stably, providing an online scheduling feedback management platform for UAV remote sensing network.
\end{abstract}

Keywords: JBPM Engine, Petri nets, emergency dispatch management, UAV remote sensing network.

\section{Introduction}

Recent years, with its flexibility and low requirement for flight security, UAV has played an important role in emergency response and spatial data. It is gradually becoming an indispensable high technology for emergency response. China is large and liable to disasters, which always happen randomly both in space and in time, but our UAV remote sensing resources are not enough to response for all the emergency tasks ( $\mathrm{Li}$ Deren et al, 2014; Li Chuanrong et al, 2010). Therefore, one optional solution for this situation is to manage the UAV remote sensing resources at a national level, and build a distributed UAV remote sensing network. Based on the platform for the system of national spatial data acquisition and application and data sharing, we can realize it.

In order to realize the above mentioned objective, and also make the UAV remote sensing network standardized and efficient, it is necessary to establish an emergency dispatch management system for UAV remote sensing network. This paper established a hierarchical model for the emergency dispatch management system for UAV remote sensing network based on the JBPM workflow engine and Petri net theory. Then an emergency dispatch management system is developed.

According to emergency dispatch of UAV remote sensing network management procedures and standard dispatch management, the system can reduce the subjectivity and the amount of human work, thus increase the efficiency and order of the emergency dispatch system. In addition, the system can also provide process custom services, and deal with the renewal and development of the emergency dispatch management system for UAV remote sensing network without modifying the software code.

\section{Workflow and the Technological Overview of JBPM}

\footnotetext{
Corresponding author. Tel.: + 86-01082178616; fax: + 86-010-82178638

E-mail address: crli@aoe.ac.cn.
} 
Workflow is a computer oriented model of operation processes. It is the business model operated by the workflow management system, and can be integrated and automated in the computer environment (Fan Yushun, 2001). Java Business Process Management (JBPM) is a widely used open source workflow engine. It is a light weighted workflow management system based on J2EE, part of or all of its operation process can be automated in a computer application environment (http://www.jboss.org/jbpm).

The three main advantages of JBPM are as follows:

1) The service logic of JBPM employs jPDL process description language of its own, other than some of the current standards. jPDL takes a workflow as an improved UML state diagram. It employs an illustrative process language to show the business process graphically. The state diagram is defined in more detail, which is easier to understand.

2) JBPM is utilized by Java language, its core functions are packaged into a simple Java library. It provides an external interaction API set with plenty of functions, on which one can expand its functionality.

3) JBPM employs Hibernate to manage the database. As one of the best data storage layer solutions in Java, Hibernate supports the mainstream database very well. By using Hibernate, JBPM leaves out the data management and focuses on the business logic (Jia Dinan, 2010).

The framework and implementation process of JBPM is shown in Fig. 1. Here is the basic working steps of JBPM:

1) Define the process by the jPDL graphical process editor, and load it into the application;

2) Start the process, that is, to create a process instance;

3) Execute the interface so that the user and system can operate the process instances, thus promote the circulation process;

4) Monitoring the process, acquiring the exact status of the running process and the to do list.

The JBPM implementation interfaces ensure that the process instance is accessible by the user and the system. Users can get the to-do tasks by the access to the task interface. In JBPM framework, the workflow engine is the core module, whose main function is to interpret the process file defined by jPDL, provide the runtime environment for process instances, dispatch the implementation of process instances, and provide interfaces of process monitoring which are provided by the API (Shang Hongjie, 2009; Fu Ming et al, 2008).

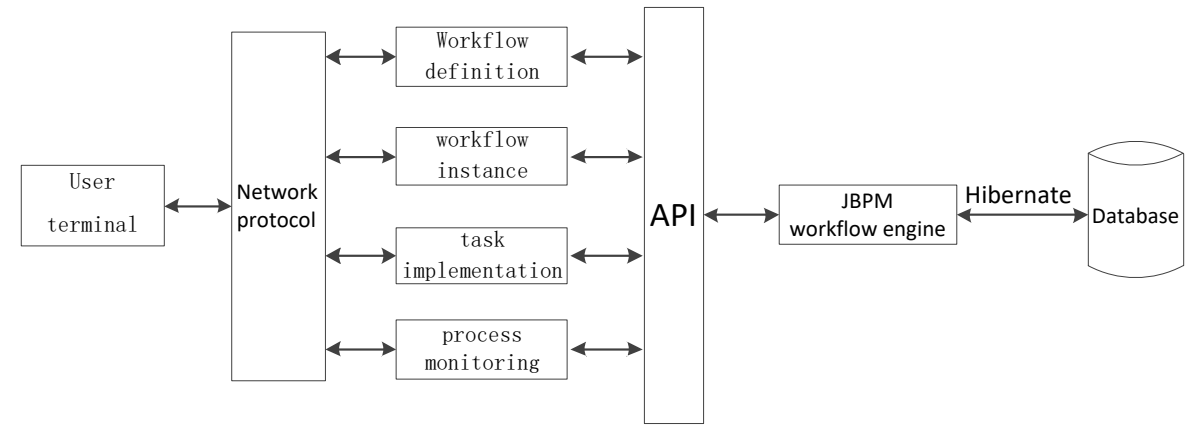

Fig. 1: JBPM framework and implementation.

\section{System Modelling and Verification Based on Petri Net}

\subsection{System Modelling}

Workflow model is the illustration of computerization of business process. There are various modelling method of workflow, such as the modelling method based on network activity, UML activity diagram and Petri nets. Since Petri net has not only graphical features that can be easily understood, but also a solid mathematical basis, it is a powerful tool for process modelling and analysis. In addition, you can also use its structure to test the validity of the model (Yuan Chongyi, 2005), so in this paper, we will use Petri network as the system modelling method.

Fig. 2 is a work flowchart of emergency dispatch management system for UAV remote sensing network. The initialization of the workflow is the application of a starter who gives the task information; then the staff 
of the emergency command and dispatch center analyze the remote sensing payload, UAV resources, flight routes and support conditions in the mission area, forming the task analysis report. Subsequently, they plan the task according to the task analysis report, establish the emergency dispatch team, and prepare to start the task while the related executor update the technical status of the system. The staff prepare the emergency response task book based on the reports updated by the related executor, hand it in to the decision makers for review. If the task book is not approved, it will be returned to the system staff to revise; if the task book is approved, the task book is assigned to each related participant. Each participant feedback the feasibility assessment results of the task book. If any of the participants has a problem, then the problem is found out and task book revised. If there is no problem, then execute system transition according to the task book, make emergency response implementation plan, and prepare the on-site technics. After the preparation works are completed, emergency response flight missions are executed by UAVs, and data of the disaster area are acquired by remote sensing. Then the data is transferred immediately to the emergency dispatch center. The professionals will make an emergency situation assessment report to the decision-making departments according to the data and other information, then give the data and the assessment to the applicant. Finally, the data from remote sensing is cataloged and archived, the performance of the task is reported and then the workflow ends (Li Chuanrong, 2014).

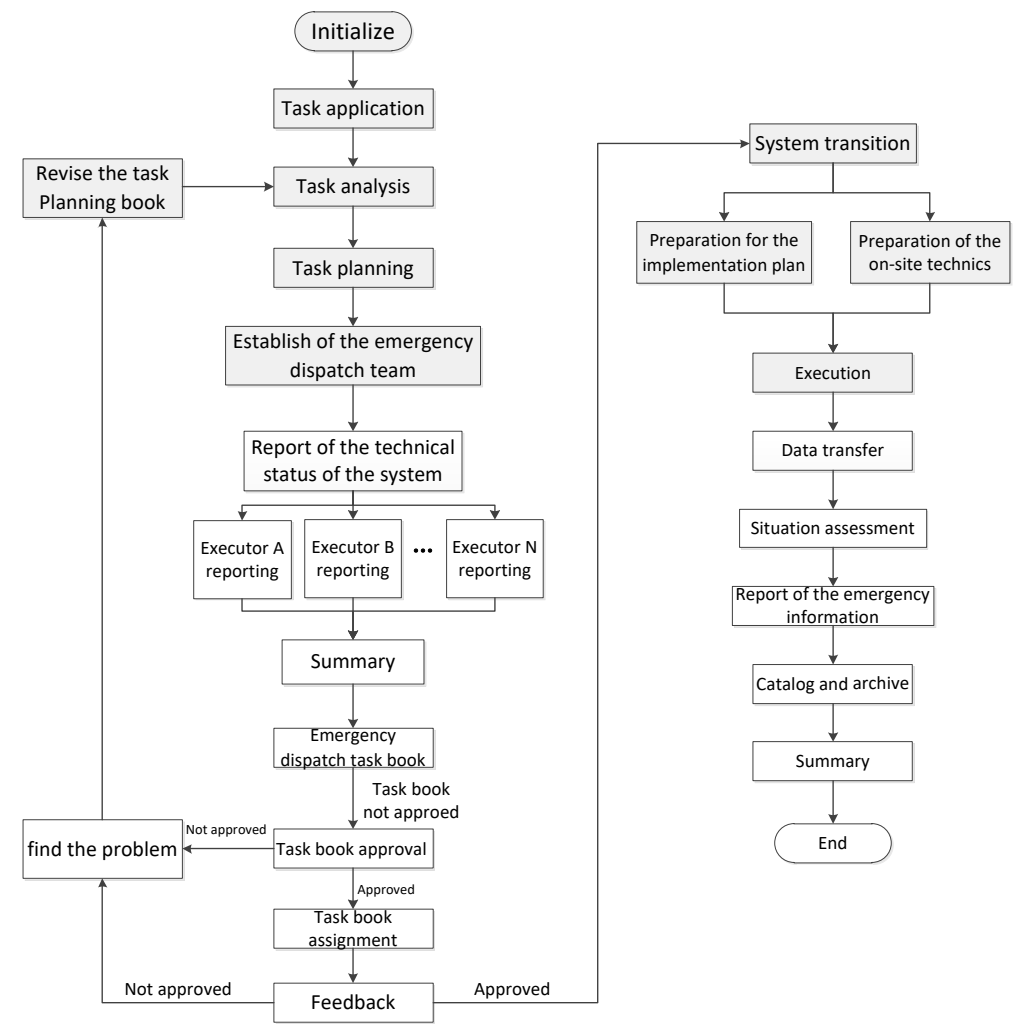

Fig. 2: Workflow model of the emergency response dispatch management system

Petri net is a directed graph made up of node and arcs. It has two types of nodes: one is called Library, usually denoted by a circle, represents the location, status, condition, and so on, another is called Change, denoted by a square or a thick bar, represents the task of the workflow. The connection between the two types of elements is denoted by a directed arc, represents the logical confection of the workflow. And the library can only be connected to changes, vice versa. Another important element of Petri net is Token, which represents the condition, resource, and status of the system.

By model transformation, the emergency dispatch management workflow model for UAV remote sensing network can be transformed into a Petri net model, as shown in Fig. 3. The superscript of the circle indicates the name of the Library, and the superscript of the flat rectangle represents the name of Changes. 


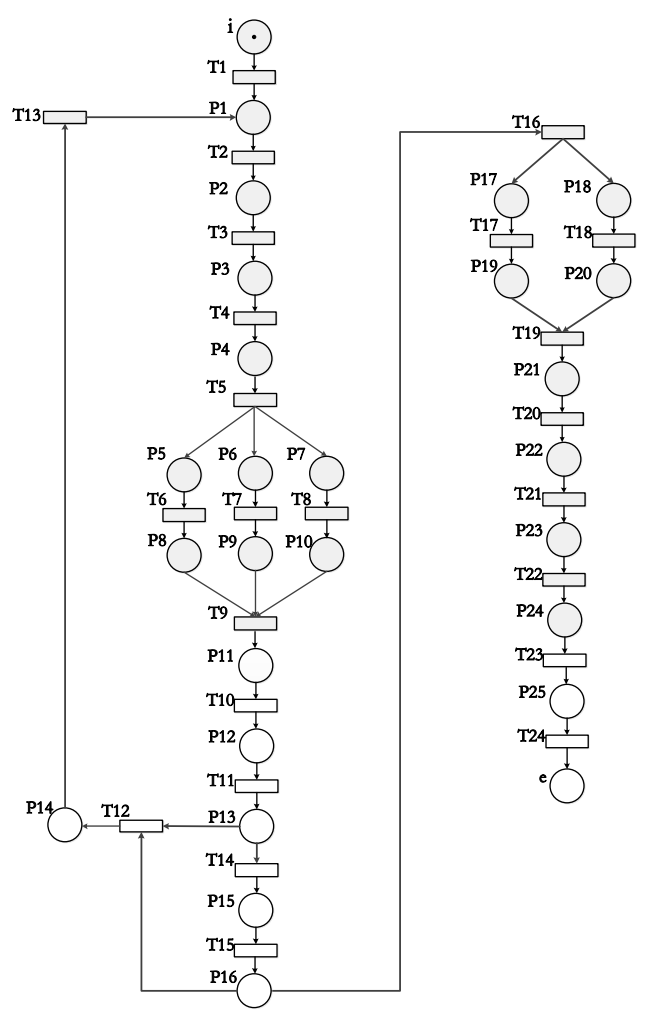

Fig. 3: Petri net model of the emergency response dispatch management system for UAV remote sensing network

The meaning of the Changes in the diagram is shown in Table 1.

Table 1: Change sets in the Petri net model of the emergency response dispatch management system

\begin{tabular}{|c|l|c|l|}
\hline Changes & \multicolumn{1}{|c|}{ meaning } & Changes & \multicolumn{1}{|c|}{ meaning } \\
\hline $\mathrm{i}$ & initialization & $\mathrm{T} 13$ & Revision of the task book \\
\hline $\mathrm{T} 1$ & $\begin{array}{l}\text { application for emergency task of the UAV } \\
\text { remote sensing network }\end{array}$ & $\mathrm{T} 14$ & Approved: task book assignment \\
\hline $\mathrm{T} 2$ & report of the task analysis & $\mathrm{T} 15$ & Feedback \\
\hline $\mathrm{T} 3$ & Assign the task plan by task requirements & $\mathrm{T} 16$ & System transition \\
\hline $\mathrm{T} 4$ & Establish of the emergency dispatch team & $\mathrm{T} 17$ & implementation plan \\
\hline $\mathrm{T} 5$ & Report of the technical status of the system & $\mathrm{T} 18$ & Preparation of the on-site technics \\
\hline $\mathrm{T} 6$ & technical status report by executor A & $\mathrm{T} 19$ & Flight and data acquisition \\
\hline $\mathrm{T} 7$ & technical status report by executor B & $\mathrm{T} 20$ & Data transfer \\
\hline $\mathrm{T} 8$ & technical status report by executor N & $\mathrm{T} 21$ & rapid situation assessment \\
\hline T9 & Summary of the status report & $\mathrm{T} 22$ & Report of the emergency information \\
\hline T10 & Emergency dispatch task book & $\mathrm{T} 23$ & Catalog and archive \\
\hline T11 & Emergency dispatch task book approval & $\mathrm{T} 24$ & Summary \\
\hline T12 & find the problem if not approved & $\mathrm{e}$ & End \\
\hline
\end{tabular}

\subsection{Simplification and verification of the model}

Petri net not only has provided a formalized representation methods for system modelling, but also has rich methods of analysis and validation, such as algebra analysis method based on the equation of state, chart analysis method based on the accessibility and inductive analysis method based on the reduction, etc. The rationality of a workflow process means that the process of workflow modelling is correct, and there will not be a deadlock, or a wrong running result (Gheng Jianjun, 2009).

As shown in Fig. 3 , the main basis for the verification of the rationality of Petri net model of the emergency dispatching management system for UVA remote sensing network is shown as follows: 1) The 
model must have a start and an end Library, and the end Library can only be reached by the start Library. Every Library and Change is between the start Library and the end Library; 2) In any case, the workflow can end, and when it ends, there exists only one Token in each instance in the end Library. And there is no Token of the same instance in other Library; 3) There is no dead Change in the Petri net, and every Change may be executed.

This paper utilizes the simplification analysis method to analyse the rationality of the model. This method can reduce the model size to an appropriate scale and reduce the complexity of the verification while maintaining the characteristics of the model. In addition, if there are problems such as deadlock or structural conflict in the workflow process of the model, they are easily detected in the graphical simplification process.

The simplification analysis method is shown in Fig. 4. This method can simplify the free-choice extended workflow net, which is active and bounded, into a closed loop network which has only one Library and Change in a limited time. Or it simplifies the workflow net into a simple sequential structure so as to complete the rationality verification of the model quickly.
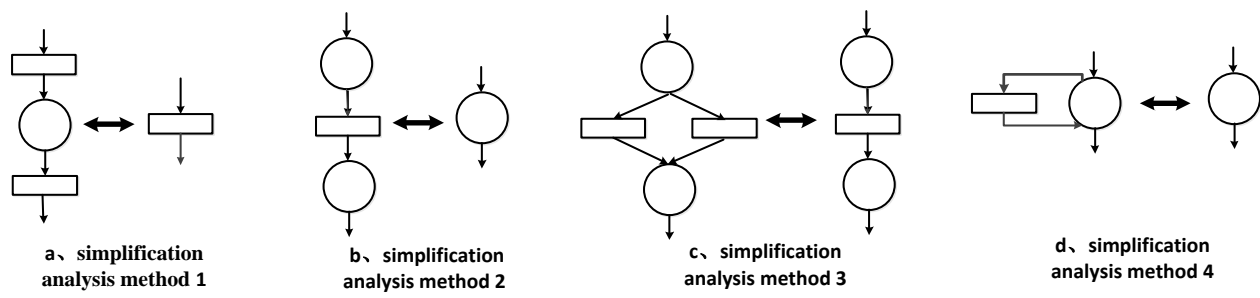

Fig. 4: simplification rules for the Petri net model

According to the validation method of the rationality of the Petri net model, we can validate the Petri net model of emergency dispatching management system for the UAV remote sensing network. First of all, we simplify the model according to the simplification rules. The model made in the process is shown in Fig. 5.

1) According to simplification rule No.1, we can eliminate Library P2、P3、P4、P5 and merge Change T2、T3、T4、T5 into T2-5. Similarly, we can eliminate Library P11、P12、P15、P21、P22、 P23、P24、P15 and get a simplified model WF1;

2) According to simplification rule No.2, we can eliminate Change T6、T7、T8, T17、T18, and divide the simplification rule No.3. P5、P6、P7 are parallel structures, and we can simplify them and the pre-Change T2-5 and the post-Change T9 into one Change T2-9. Similarly, we eliminate the parallel structure made of T17 and T18 and get a simplified model WF2;

3 ) According to simplification rule No.3, we can eliminate Library P14. According to simplification rule No.2, we can eliminate Change T14-15 and T2-11 and get the simplified model WF3;

4) According to simplification rule No.4, we can eliminate Change T12-13. According to simplification rule No.1, we can eliminate Library P13 and get the final model WF4 with simplified structure.

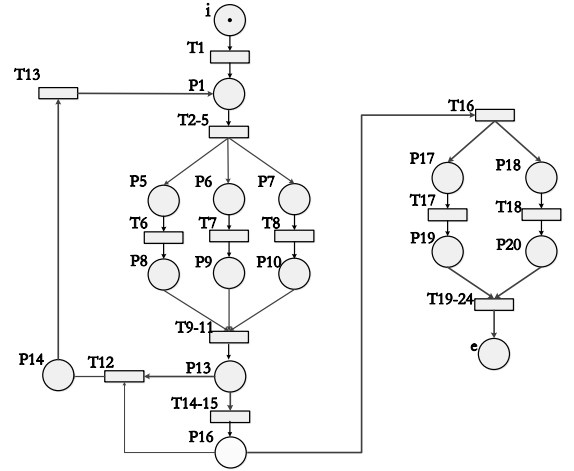

(a) Model WF1

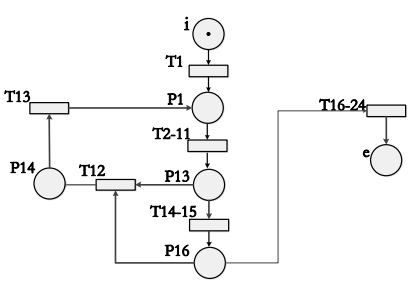

(b) Model WF2

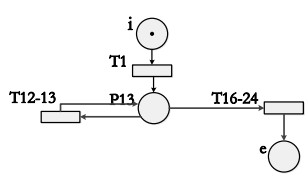

(c) Model WF3

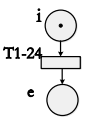

(d) Model WF4

Fig. 5: Simplification of the Petri net model

By the simplification process, we can simplify the Petri net model of the emergency dispatching management system for the UAV remote sensing network (as shown in Fig. 3) into a simple sequential structure. We can conclude that the workflow Petri net model meets the requirement of rationality. 


\section{Design and realization of the system}

\subsection{Structure design of the system}

According to the characteristics of the emergency dispatching management system for the UAV remote sensing network, this paper divides the frame structure of the emergency dispatching management system into three layers: the application layer, the service layer and the data layer. The frame structure of the system is showed in Fig. 6.

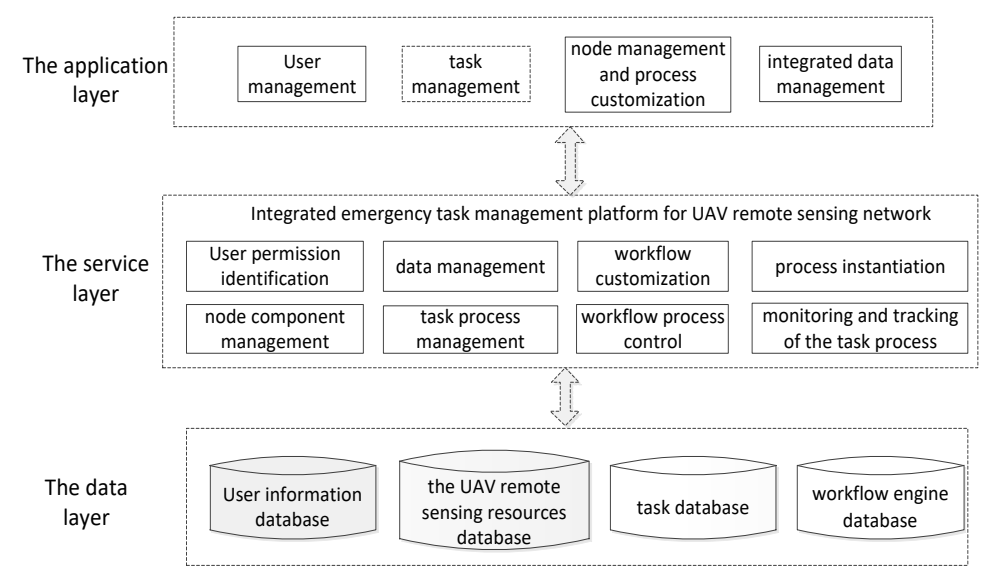

Fig. 6: Frame structure of the emergency dispatching management system

The application layer is mainly designed to provide the participants, who are involved in the emergency dispatching management system for the UVA remote sensing network, with operation functions, including user management, task management, node management, process customization and integrated data management. Emergency dispatching management participants include system administrators, task applicants and reviewers, etc.

The service layer is the core of the system. It mainly provides basic services for the emergency dispatching management system for the UAV remote sensing network which is based on workflow. The services include user permission identification, data management, node component management, task process management, workflow customization and workflow process control, process instantiation and the monitoring and tracking of the task process, etc.

The data layer is the support layer of the system data. It provides data access service which is mainly based on the relational database. All the data is divided into user information database, the UAV remote sensing resources database, task database and the workflow engine database and so on.

\subsection{Structure design of the system}

In this paper, the emergency dispatching management system for the UAV remote sensing adopts B/S structure. The whole system is developed by Myeclipse and development language Java and JSP. The workflow engine utilizes JBPM4, and the background database Oracle, and the client of the system is the mainstream browser software.

According to the different identities of the participants in the emergency dispatching management system for the UAV remote sensing network, the administrator can define different names for different characters, whose operation authority is different. When applying for a task, this system selects node components which represents the emergency dispatch management workflow model from workflow engine by visual methods. Then the task workflow template is made by the workflow model. Afterwards, task applicants apply for the instantiation workflow template of the emergency task for UAV remote sensing network, while the system records the ID of the task process instance backstage. Then it stores the information of the instantiated task in the tasks database through the task process management service, and automatically relates the information with task workflow template. Subsequently, based on the current workflow templates, the status of the workflow node and the input data, the workflow engine realizes the flow of the work process node according 
to predefined rules. Finally, the whole process management of the emergency dispatching for UAV remote sensing network is realized.

According to the previous established model, the system provides customers with the task application interfaces of the emergency service for UVA remote sensing network. After receiving the tasks application information, task book nodes help users decide the remote sensing load of a mission, the UVA resource, flight routes and supporting conditions, et al. Afterwards, the requirements of task participants is formulated, and the task book is assigned to the participants. The participants give feedbacks on their respective tasks. The nodes of the system state include summary and report of the system state by all of the participants. The system displays the execution status and its trend of the tasks by graphical methods, and plans the task trends and the routes according to the characters of each task node. It also has the function of completion and playback, etc.

\section{Conclusion}

Utilizing various illustrative ways such as text and charts, this article developed an emergency dispatching management system for the UAV remote sensing network based on JBPM. The currently famous light-weighted workflow technique is used. The system provides an online dispatch platform with interactive feedback for the operation of UAV remote sensing network. Compared with the normal task management system, this paper provides a visual management platform based on workflow. It not only displays the business process and the running state of the emergency dispatching management system for the UVA remote sensing network directly to the user, but also realizes the overall lifecycle management of the emergency dispatching management system for the UVA remote sensing network, providing users with an intuitive environment which is convenient to operate and maintain. At the same time, the system provides the workflow customization function. Customers can update their emergency dispatching management workflow model according to the evolution of the emergency business process. It only needs the development of new key component management node, which reduces the coupling level of the system and the actual service logic. The operation results show that the system has achieved the expected performance, which has better versatility, extensibility, stability, and the adaptability to the business logic evolution. However, the system is centralized, which has lower integration and security. In the future research, the function of the workflow system is modularized by the distributed strategy. Through the coordination between the various modules, it can achieve the intended function.

\section{Acknowledgment}

Project supported by the National High-tech R\&D Program of China (863 Program) (No. 2013AA122904; No. 2013AA122103).

\section{References}

[1] Li Deren, LI Ming. Research Advance and Application Prospect of Unmanned Aerial Vehicle Remote Sensing System. GEOMATICS AND INFORMATION SCIENCE OF WUHAN UNIVERS, 2014, 39(5): 505-513.

[2] Li Chuanrong, Wang Chaoliang, Liu Junxian. Spatial data Acquisition and Application Coordination System for Emergency Response. National Comprehensive Disaster Prevention and Mitigation and Sustainable Development Forum, 2010:100-104

[3] Fan Yushun, Workflow management technology. Tsinghua University press, 2001.

[4] http://www.jboss.org/jbpm

[5] Jia Dinan. Design and Implementation of Office Systems Customizable Based on JBPM Process. Beijing University of Post and Telecommunication. 2010.

[6] Shang Hongjie. Design and Implementation of Surveying and Mapping Production Management System Based on JBPM . Lanzhou University, 2009.

[7] Fu Ming, Zhang Wei. Open source J2EE - based Workfiow Engine JBPM Design and Implementation. Computing Technology and Automation, 2008, 27(4): 111-114. 
[8] Yuan Chongyi. Principle and application of Petri net. Electronic Industry Press, 2005:32-178.

[9] Li Chuanrong. The Technology of Unmanned Aerial Vehicle Remote Sensing System. Science Press, 2014.

[10] Gheng Jianjun. Graduate cultivation workflow model based on Petri nets. Journal of Hefei University of Technology, 2009, 32(1): 78-81. 